\title{
POSSIBLE EVIDENCE FOR A BINARY MASSIVE BLACK HOLE IN THE GALACTIC NUCLEUS
}

\author{
E.J.A. MEURS \\ Dunsink Observatory \\ Castleknock, Dublin 15, Ireland
}

\begin{abstract}
The Galactic Centre candidate Sgr A* may exhibit a $40 \mathrm{~km} / \mathrm{s}$ radial velocity component, which is not observed for $\mathrm{OH} / \mathrm{IR}$ stars around the centre. This could be interpreted as orbital motion of one member of a binary massive black hole. In other galaxies such pairs may be inferred from radio jet precession and emission line profiles.
\end{abstract}

\section{The Galactic Centre}

The intricate radio source $\mathrm{Sgr} \mathrm{A}$ West is commonly considered as the centre of our Galaxy (e.g. Genzel 1987). Within this region, the unique, strong radio source $\mathrm{Sgr} \mathrm{A}^{*}$ is the main candidate for a central black hole (cf. Lo 1987). Mass estimates for such an object have been derived from the overall emission properties of Sgr A* (for example, Melia et al. 1992, Ozernoy 1995) and from the inferred mass distribution in the Galactic Nucleus (for example, Townes 1995). The available mass determinations appear to separate between either $\sim 10^{6} \mathcal{M}_{\odot}$ or $\sim 10^{2} \mathcal{M}_{\odot}$.

\section{Binary Black Holes in the nuclei of galaxies}

Theoretical modelling of galaxy mergers suggests that pairs of massive black holes may dwell in the centremost region of galaxies, as a result of past mergers. Their masses probably depend on the previous history of the core in each of the merging galaxies. Normally they would stay at a distance of a few parsec apart, until a next merger takes place. Then the separation of the two black holes may decrease, followed by coalescence, after which a 
new pair of black holes is established with the incoming galactic nucleus. This scenario is described more in detail by Roos (1988).

The close location of two such black holes renders it difficult to recognize the binarity when other galaxies are concerned. VLBI might detect this if both black holes would emit radio radiation. Otherwise, more indirect methods will stand a better chance. One example of this is the temporal variability pattern of suitable optical emission lines (Halpern \& Filippenko 1988). An appropriate indication for binary compact objects is also the precessional behaviour of radio jets (e.g. Rees 1984). How the dynamical evolution of a binary massive black hole may be reflected in a changing precession period is elaborated by Roos (1988) and Roos et al. (1993).

\section{Possible evidence for binarity in the Galactic Centre}

Of course, the centre of our Galaxy offers the best spatial scale to search for binary compact objects. An intriguing observation exists (see Clube 1986) of the radial velocities of two $\mathrm{H}_{2}$ emission regions straddling the Galactic Centre (a likely torus shaped geometry). The observed radial velocity asymmetry between these two peaks implies a radial velocity component of 40 $\mathrm{km} / \mathrm{s}$ with respect to the sun. Yet, no such difference is noticeable from the distribution of radial velocities of $\mathrm{OH} / \mathrm{IR}$ stars around the Galactic Centre (measured from radio maser lines). These objects show evidence of rotation around the Centre, but have essentially no offset in radial velocity (Habing 1995). An alternative interpretation of the $\mathrm{H}_{2}$ radial velocity asymmetry would be that the observed difference originates from the orbital motion of one member of a binary massive black hole. The apparent dichotomy in the mass estimates for a central compact object (see Section 1) could find a natural explanation in this way.

\section{References}

Clube, S.V.M. (1986) The Observatory, 106, 166

Genzel, R. (1987) in The Galaxy, Eds R. Carswell and G. Gilmore, NATO ASI Series, p. 51

Habing, H. (1995) this volume

Halpern, J.P., Filippenko, A.V. (1988) Nature, 331, 46

Melia, F., Jokipii, J.R., Narayanan, A. (1992) ApJ, 395, L87

Lo, K.Y. (1987) in The Galactic Centre, Ed. D.C. Backer, AIP Conf. Proc., 155, 30

Ozernoy, L. (1995) this volume

Rees, M.J. (1984) AnnRevAA, 22, 123

Roos, N. (1988) ApJ, 334, 95

Roos, N., Kaastra, J.S., Hummel, C.A. (1993), ApJ, 409, 130

Townes, C.H. (1995) this volume 